\title{
Exploiting different active silicon detectors in the International Space Station: ALTEA and DOSTEL galactic cosmic radiation (GCR) measurements
}

\author{
Livo Narici ${ }^{1,2, *}$, Thomas Berger ${ }^{2}$, Sönke Burmeister ${ }^{3}$, Luca Di Fino ${ }^{1}$, Alessandro Rizzo ${ }^{1}$, Daniel Matthiä ${ }^{2}$,
} and Günther Reitz ${ }^{2}$

1 Department of Physics, University of Rome Tor Vergata \& INFN - Roma 2, 00133 Rome, Italy

${ }^{*}$ Corresponding author: narici@roma2.infn.it

2 German Aerospace Center (DLR), Institute of Aerospace Medicine, Linder Höhe, 51147 Köln, Germany

3 Christian Albrechts Universität zu Kiel (CAU), Christian-Albrechts-Platz, 24118 Kiel, Germany

Received 6 April 2017 / Accepted 19 June 2017

\begin{abstract}
The solar system exploration by humans requires to successfully deal with the radiation exposition issue. The scientific aspect of this issue is twofold: knowing the radiation environment the astronauts are going to face and linking radiation exposure to health risks. Here we focus on the first issue. It is generally agreed that the final tool to describe the radiation environment in a space habitat will be a model featuring the needed amount of details to perform a meaningful risk assessment. The model should also take into account the shield changes due to the movement of materials inside the habitat, which in turn produce changes in the radiation environment. This model will have to undergo a final validation with a radiation field of similar complexity. The International Space Station (ISS) is a space habitat that features a radiation environment inside which is similar to what will be found in habitats in deep space, if we use measurements acquired only during high latitude passages (where the effects of the Earth magnetic field are reduced). Active detectors, providing time information, that can easily select data from different orbital sections, are the ones best fulfilling the requirements for these kinds of measurements. The exploitation of the radiation measurements performed in the ISS by all the available instruments is therefore mandatory to provide the largest possible database to the scientific community, to be merged with detailed Computer Aided Design (CAD) models, in the quest for a full model validation. While some efforts in comparing results from multiple active detectors have been attempted, a thorough study of a procedure to merge data in a single data matrix in order to provide the best validation set for radiation environment models has never been attempted. The aim of this paper is to provide such a procedure, to apply it to two of the most performing active detector systems in the ISS: the Anomalous Long Term Effects in Astronauts (ALTEA) instrument and the DOSimetry TELescope (DOSTEL) detectors, applied in the frame of the DOSIS and DOSIS 3D project onboard the ISS and to present combined results exploiting the features of each of the two apparatuses.
\end{abstract}

Key words. International space station - ALTEA - DOSTEL - Galactic cosmic radiation - Space radiation measurements

\section{Introduction}

The radiation environment of relevance for deep space human exploration is generated by three different sources. The Galactic Cosmic Radiation (GCR) are generated well beyond our solar system and are the most penetrating components as they feature quite high kinetic energies. GCR are inversely modulated by solar activity (Durante \& Cucinotta 2011). The radiations associated with solar events (Solar Particle Events (SPE)) have a broad energy spectrum, but with much lower energy than GCR, and are constituted mostly by protons. These events are quite irregular in occurrence, show large spectral variations, and are statistically more frequent during high solar activity periods. These are the two primary, mostly independent, sources of radiation relevant for human exploration. The third source is the radiation due to the interaction between the primary radiation and the material between this and the astronauts (spacecraft hull, Extra Vehicular Activity (EVA) suit, etc.). Finally, the radiation trapped in the Earth's radiation belts is of no relevance for human exploration issues, but of importance when monitoring astronaut's doses in Low Earth Orbit (LEO).

The mitigation of radiation risks is an important safety issue in space travel and will have to be carefully addressed during mission planning and operations. It is foreseen that this goal will be achieved using integrated radiation models of space habitats (where we include spacecrafts). These models will be a combination of radiation source models, Computer Aided Design (CAD) models of the habitat, transport simulations and of a detailed knowledge of time variability of the first two. The development of the radiation models of space habitats is ongoing and will have to undergo a long campaign of validation against experimental data: both for the single model components (transport, source models, etc.) and for the final integrated model. Several of these validations can and should be performed on ground in particle accelerators. However, a habitat radiation model will have to take care of the extreme complexity of the shielding distribution, including its time variability due to movements of materials in the habitat, immersed in the very peculiar time 
and space mixture featured by GCR and SPE. This cannot be simulated on ground.

The International Space Station (ISS) is in this respect the best available space habitat to perform these kinds of radiation measurements. As mentioned the complexity of the spacecraft (in this case the ISS) is a problem that will have to be tackled to properly handle a habitat integrated model. The radiation impinging on the ISS is not the same as the deep space one: the Earth magnetic field provides a partial shielding against radiation. During the travel around the Earth the ISS goes through a highly shielded section of the orbit (over the equator), a much less shielded section (at high latitudes), and a particular region, over Brazil, named South Atlantic Anomaly (SAA), where the Earth's radiation belts get closer to the Earth and trap a large amount of low-energy protons generating a total radiation spectrum quite different from the deep space one. The SAA is indeed a significant deviation from the deep space radiation environment in LEO. The capability of active detectors to follow the time dynamic of the radiation during the orbital path permits to select out SAA, therefore allowing for constructing a radiation database with a spectral content that best mimics what will be found during deep space travel.

A number of radiation measurements have been performed in the ISS with active detectors Narici et al. (2015b). No full attempt has been done so far to merge space radiation data coming from different detectors in a single dataset, taking into account the properties of the systems used. Detector simulation models, triggering and saturation issues can be studied and the data corrected accordingly before merging results from different devices in a single dataset. Even though these pre-analyses will increase data uncertainties, the merged dataset will be a quite powerful tool for model validation.

In this paper we study the performance of two active detector systems DOSTEL (DOSimetry TELescope) and ALTEA (Anomalous Long Term Effects in Astronauts) that measured radiation in the ISS between 2010 and 2012 and in the case of DOSTEL are still measuring to date. A merged dataset is built, and common results, in flux and dose rates, are discussed. The procedure that made this merging possible is described in detail.

\section{Description of the detectors}

\subsection{ALTEA}

The ALTEA system has been developed for a series of measurements in the ISS, initially devoted to the study of the perception of visual illusions by the astronauts in orbit (Narici 2008). The detector system is a set of six telescopes. Each telescope (hereafter SDU: Silicon Detector Unit) features six sensitive silicon planes, each striped in alternate directions " $X$ " and " $Y$ " and grouped in three $X Y$ pairs, spaced by $3.5 \mathrm{~cm}$ from each other. The two strip coordinates and the height of the plane pairs into the detector provide the three spatial coordinates of the striking ion. This permits to track single ion trajectories. ${ }^{1}$ Each plane ${ }^{2}$ is $16 \times 8 \times 0.038 \mathrm{~cm}^{3}$. Each SDU is auto-triggered by ions passing through the first and fifth plane and delivering a LET (Linear Energy Transfer)

\footnotetext{
${ }^{1}$ The trigger and nature of the detector does not allow discrimination between forward and backward moving particles.

${ }^{2}$ Each plane is made by two striped silicon chips mounted side by side.
}

of $3 \mathrm{keV} / \mu \mathrm{m} \leq \mathrm{LET}(\mathrm{Si}) \leq 700 \mathrm{keV} / \mu \mathrm{m}$. As will be seen in detail, this lower cut leaves out most protons and He ions. The double-directional Geometrical Factor (GF) is $230 \mathrm{~cm}^{2}$ sr (calculated from our Monte Carlo - PHITS (Particle and Heavy Ion Transport code System) ALTEA model, and consistent with Sullivan 1971). Further details of the instrument can be found in Zaconte et al. (2008). Data transfer from ISS to ground is in real time and is described in Di Fino et al. 2006. Under certain conditions it is possible to estimate the charge of each impinging particle (Di Fino et al. 2012). During the investigation National Aeronautics and Space Administration (NASA) provided a set of orbital ancillary data that permitted to select measurements in different geomagnetic zones (Zaconte et al. 2008, 2010a, 2010b).

\subsubsection{ALTEA: data pre-analysis}

The raw data underwent first a subtraction of the pedestal (offset with no input, measured from non-hit silicon chip stripes) in order to calibrate the baseline of each silicon chip. Information for pedestal subtraction is provided periodically throughout the measurements to take into account possible drifts during the measurements. To minimize the noise contribution only events showing an aligned single track are selected, leaving out spurious events (most likely noise) and multiple particle tracks due to possible showers most likely caused by fragmentations in the detector system or in the nearby rack instruments. The most valuable information obtained by the measurements is the energy released in each silicon plane (and strip) by each ion $(\Delta E)$ and the angle between the trajectory and the normal to the silicon plane $(\theta)$. The LET in silicon for each ion is therefore given by $\operatorname{LET}(\mathrm{Si}) \approx \Delta E /(h \cos \theta)$, where $h$ is the silicon thickness $(380 \mu \mathrm{m})$.

In this work for each particle we use the average deposited energy over the six planes in each telescope and when two SDUs are stacked in the same directions ( $X Y Z$ configuration, see below) the average over the two SDUs. We analyzed the data to obtain flux and dose rates. ALTEA is a telescope and therefore measures the radiation impinging on it within its field of view. To calculate these radiation quantities we assume an isotropic source and use the geometrical factor (GF) to estimate the best source that would have caused the obtained measurements, and finally use this source to calculate the radiation quantities of interest.

\subsubsection{Flux}

The flux (particle rate per area and steradian) is therefore obtained simply dividing the counting rate by the geometrical factor.

\subsubsection{Dose rate}

The dose rate is the energy per mass deposited in a time interval. As mentioned ALTEA is a telescope and does not measure all ions impinging on its sensitive volume. It is possible to estimate that for each measured particle there are 1/GF particles impinging on the detector per steradian per unitary area. So that (see Eq. (1)):

$$
\text { Dose rate }(\mathrm{Si})=\frac{4 \pi}{h \rho_{\mathrm{si}} \mathrm{GF}} \frac{1}{\Delta t} \sum_{k=1}^{N} \Delta E_{k},
$$

where GF is the geometrical factor, $\rho_{\mathrm{Si}}$ is the Silicon density, and the sum is extended to all the $N$ particles measured in $\Delta t$. The $4 \pi$ coefficient comes from the integration over the full 
Table 1. Timing of the overlap measurement periods, with the indication of the ALTEA positions. The total duration can be less than the total number of days between start and end because of shut-off periods.

\begin{tabular}{|c|c|c|c|c|c|}
\hline No & From & To & ALTEA site & Full days & For each ALTEA site \\
\hline 1 & 20-Sep-2010 18:58:00 & 02-Oct-2010 03:25:00 & P1: Lab1S1 & 11 & 11 \\
\hline 2 & 15-Oct-2010 17:32:00 & 25-Oct-2010 11:14:00 & P2: Lab1O2 & 09 & \\
\hline 3 & 31-Oct-2010 12:33:00 & 30-Nov-2010 09:48:00 & P2: Lab1O2 & 29 & 38 \\
\hline 4 & 24-Apr-2011 15:12:00 & 17-Jun-2011 14:54:46 & P3: Lab1P4 & 53 & 53 \\
\hline 5 & 21-Мay-2012 11:28:54 & 23-May-2012 00:08:00 & P4: Lab1S6 & 01 & \\
\hline 6 & 24-May-2012 00:00:00 & 08-Jun-2012 07:00:00 & P4: Lab1S6 & 14 & 15 \\
\hline 7 & 08-Jun-2012 12:05:00 & 19-Jul-2012 04:07:00 & P5: Col ER3 & 40 & \\
\hline 8 & 20-Jul-2012 12:19:00 & 20-Jul-2012 18:11:00 & P5: Col ER3 & 00 & \\
\hline 9 & 27-Jul-2012 08:05:00 & 04-Aug-2012 03:58:00 & P5: Col ER3 & 08 & \\
\hline 10 & 04-Aug-2012 14:30:00 & 08-Aug-2012 23:59:00 & P5: Col ER3 & 03 & \\
\hline 11 & 09-Aug-2012 18:08:00 & 30-Sep-2012 14:35:00 & P5: Col ER3 & 51 & 102 \\
\hline Total & 20-Sep-2010 18:58:00 & 30-Sep-2012 14:35:00 & $\mathrm{P} 1-\mathrm{P} 5$ & 219 & \\
\hline
\end{tabular}

solid angle. To avoid introducing further uncertainties in the analyses of the two detectors (ALTEA and DOSTEL), we will use throughout the paper dose rates in Silicon. The conversion to dose rates in water can be readily done using one of the strategies proposed in the literature (see e.g., Benton et al. 2010).

The isotropy assumption requires further comments. It is obviously not valid during passages through the South Atlantic Anomaly (SAA), and, furthermore, the presence of the Earth shielding should be accounted for when considering the contribution of the Galactic Cosmic Radiation (GCR). The former issue (SAA) is mitigated by the directional feature of the detectors: the isotropy assumption is required for each telescope. When reconstructing the source from one telescope measurement (see above) it will refer only to the direction of that telescope.

The latter issue (Earth shadow) reduces the number of particles measured by ALTEA (more so in the $Z$ direction for a factor $\approx 1 / 2$ ). Our procedure takes this into account. If explicitly considering the Earth shielding the result would indeed not change: for example for the $Z$-directed telescopes we would have used a single ended GF (which is $1 / 2$ of the double ended), assuming all particles coming from above, then integrate only over $2 \pi$, producing the same result (Narici et al. 2015a).

\subsubsection{ALTEA: configurations}

During the periods of concurrent ALTEA and DOSTEL measurements, ALTEA was moved to four different positions in the USLab and to one position in Columbus. The exact overlap periods, with the indication of the ALTEA position, can be found in Table 1 and are given in graphical representation also in Figure 1.

During the acquisitions in the USLab, ALTEA was deployed in the so-called $X Y Z$ configuration. In this case, an ad hoc plate system holds the six ALTEA SDUs stacking two SDUs in each of the Cartesian directions (see Fig. 2). Care has been taken to position ALTEA each time with these three directions parallel to the main Station axes. During the last period in Columbus, only a single SDU, Z-directed, was used, and this was inserted in an empty double drawer in ER3 in Columbus.

\subsubsection{ALTEA: positions}

In position 1 the ALTEA $Y$ direction was inserted in a missing rack space in the starboard side of the USLab; in position

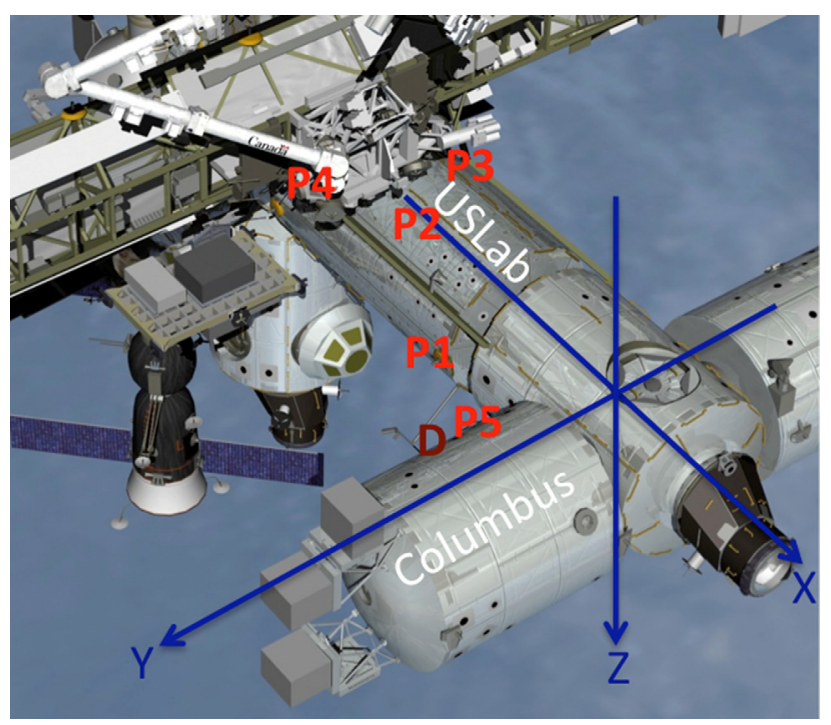

Fig. 1. The five positions of ALTEA (P1-P5) and the position of the DOSTEL (D) instruments, with the indication of the three Station axes (from Narici et al. 2015a).

2 ALTEA $Z$ direction was inserted in an empty drawer in the overhead side of the USLab; in position 3 ALTEA $Y$ direction was inserted in an empty drawer in the port side of the USLab; in position 4 ALTEA was positioned at the corner between overhead and starboard side, external from the racks; in position 5 the only active SDU (Z-directed) was inserted in an empty double drawer in ER3 in Columbus (see also Table 1 and Fig. 1). Note: refer to Figure 1 to estimate the positions relative to the ISS structures.

\subsection{DOSTEL}

DOSTEL has been and is currently used in the frame of the experiment Dose distribution within the ISS (DOSIS, 20092011) and the DOSIS 3D (since 2012, ongoing) project onboard the Columbus Laboratory for the long-term determination of the radiation field parameters at a fixed location inside Columbus. For this project two DOSTEL units are applied (see also Fig. 3).

Each DOSTEL includes two passivated implanted planar silicon (PIPS) detectors $(315 \mu \mathrm{m}$ thick, with an active area of $6.93 \mathrm{~cm}^{2}$ ) arranged in a telescopic geometry (distance 


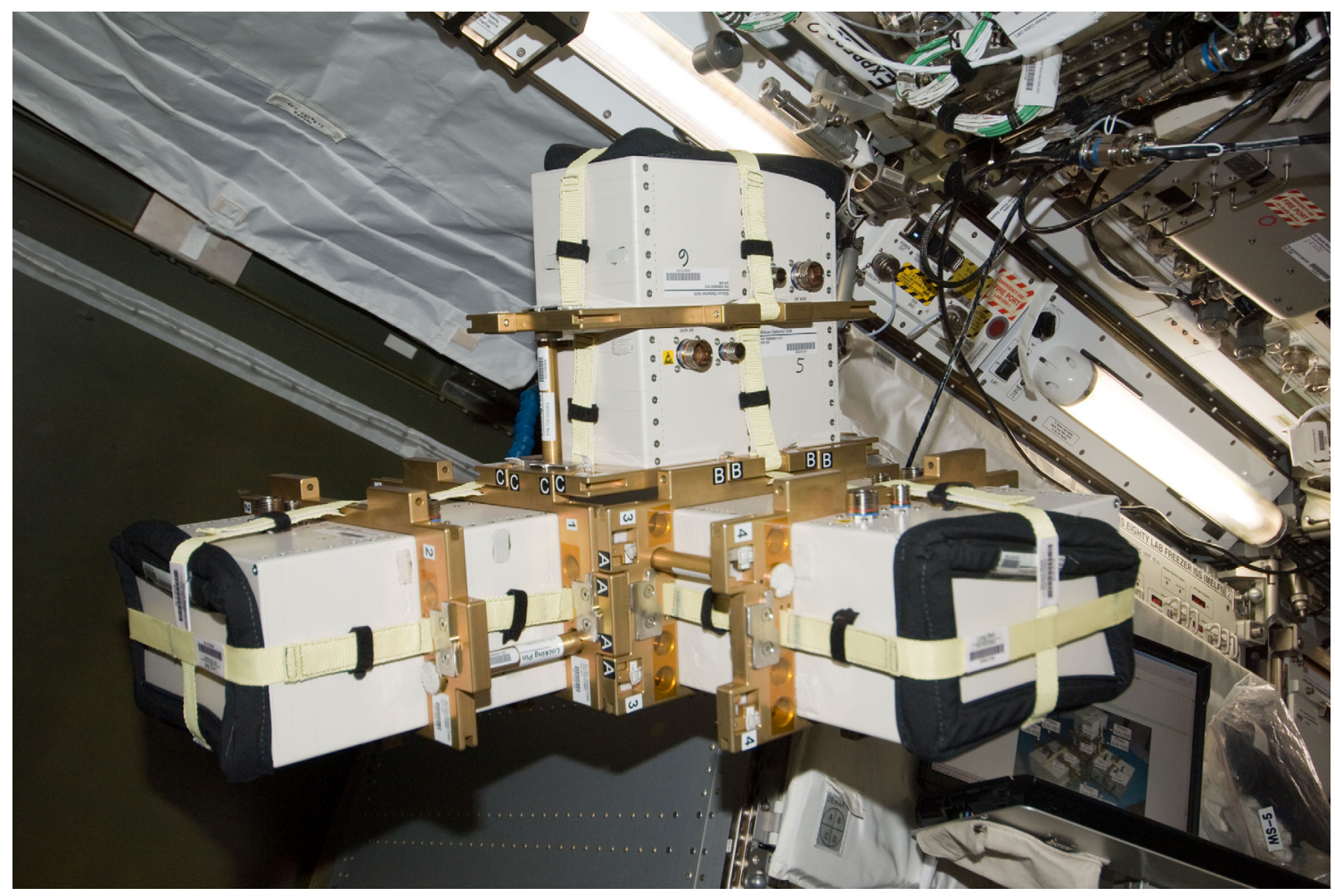

Fig. 2. The ALTEA $X Y Z$ configuration as deployed in a measurement site. Source: NASA/ESA.

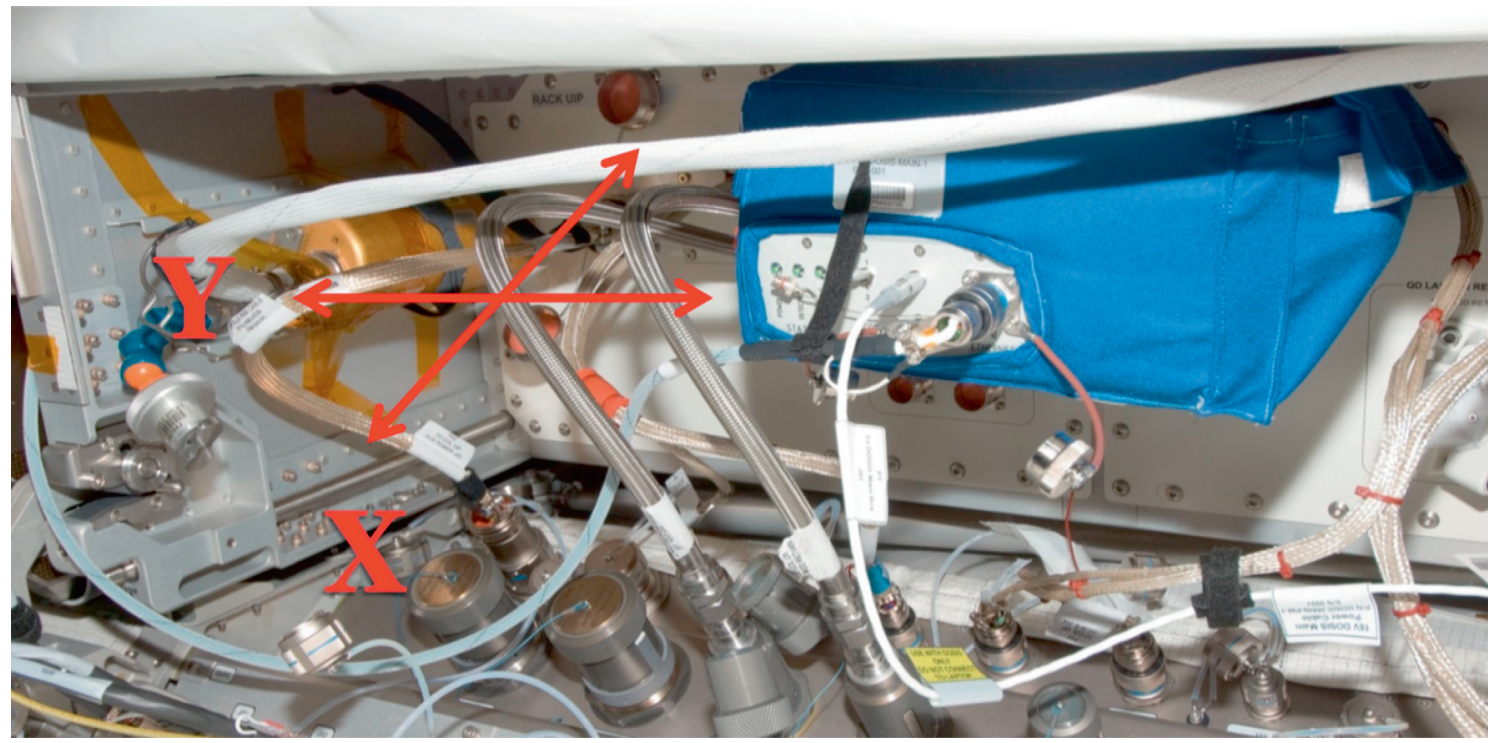

Fig. 3. The two DOSTEL units positioned inside the DOSIS-MAIN-BOX (Blue Nomex pouch) beneath the European Physiology Module (EPM) in Columbus. Shown is also the viewing direction of the DOSTEL-1 instrument $(X)$ and the DOSTEL-2 instrument $(Y)$. Source: ESA/ NASA.

between detectors: $1.5 \mathrm{~cm}$, full opening angle of $120^{\circ}$, doubledirectional geometric factor of $16.5 \mathrm{~cm}^{2} \mathrm{sr}$ ). DOSTEL measures count and dose rates due to the radiation hitting a single detector ("dose measurement mode", double-directional geometric factor: $44 \mathrm{~cm}^{2} \mathrm{sr}$ ) and coincidental hits in the two detectors with a limited path length in the detectors to derive information about the linear energy transfer (LET) ("telescope" or "LET measurement mode"). Based on the measured data DOSTEL provides absorbed dose and dose equivalent values. In this comparison with the ALTEA data only flux and dose rates will be used.

The readout electronics is different for the two detectors in the telescope, and leads to an energy deposition range in Silicon of $0.069-165 \mathrm{MeV}$ for the top detector of the telescope 
and of $0.048-66 \mathrm{MeV}$ for the bottom detector. The relevant LET range in water is therefore $0.1-240 \mathrm{keV} / \mu \mathrm{m}$ for the top and $0.07-95 \mathrm{keV} / \mu \mathrm{m}$ for the bottom detector.

Scientific and housekeeping data are downloaded via Ethernet connection nominally every four weeks. For a more complete technical description of the devices, the reader is addressed to Berger et al. (2016, 2017).

\subsubsection{DOSTEL: data pre-analysis}

The DOSTEL units measure (i) particle count rate, (ii) absorbed dose rate, and (iii) energy deposition spectra. Count and dose rates are measured by each detector of each unit independently. The dose rate is measured by summing up the Analog-to-Digital Converter (ADC) values of the relevant hits. These values measure the energy deposition in the detector, therefore the total absorbed dose deposited in a single detector is the integral of these ADC values. Particle rates and the absorbed dose rates are stored in the DOSTEL memory. The integration period is normally set to $100 \mathrm{~s}$ (GCR mode). Inside the SAA where particle rate is significantly higher, the integration period for the count rate and absorbed dose rate measurements is set to $20 \mathrm{~s}$.

In this paper we are going to consider only count and dose rates. The interested reader is addressed to Berger et al. (2016, 2017) for the procedures to calculate the LET spectra and to derive other dosimetric quantities.

\subsubsection{DOSTEL: configuration and position}

The two DOSTEL units (DOSTEL-1 and DOSTEL-2) and the DOSTEL Data and Power Unit (DDPU) are mounted, using a Nomex ${ }^{(}$pouch, beneath the European Physiology Module (EPM) in Columbus. DOSTEL-1 is directed parallel $(X)$, whereas DOSTEL-2 perpendicular $(Y)$ to the ISS flight direction (see also Fig. 1). Scientific and housekeeping data of the instruments are downlinked once a month via the EPM Rack of Columbus.

\section{Methods}

\subsection{Timeframe}

As detailed in Table 1 the comparison measurements took place in several periods between September 20th 2010, and September 30th 2012, for a total of 212 full days. Solar activity was at maximum, even if this has been the lowest solar maximum in several decades. From the NOAA (National Oceanic and Atmospheric Administration) catalog (ftp:// ftp.swpc.noaa.gov/pub/indices/SPE.txt) eight Solar Particle Events occurred in the overlap periods (most prominent: one in June 2011, two in July 2012). The altitude of the ISS ranged between about $339 \mathrm{~km}$ and $447 \mathrm{~km}$. Three significant orbital altitude increases occurred (June 2011, May 2012, and August 2012). During this period the ISS attitude was mostly constant ( $X$ axis parallel to the ISS velocity vector), brief changes in attitude occurred during the two Shuttle dockings during the overlap periods: STS $134(05 / 18 / 2011$ to $05 / 29 / 11)$ and STS $135(07 / 10 / 2011$ to $07 / 19 / 2011)$.

Unfortunately, in none of the overlap periods have the two detector systems been in the same module, at the same time, looking in the same direction. As it will be stressed in Section 5 the lack of a proper cross-calibration time for the two detectors decreases the strength of a full and detailed understanding of the comparison and pushes for a strong request for planning such comparative periods in the ISS for any detector in the future.

\subsection{Procedure}

In order to compare the sets of data coming from the two detector systems, to be able to use them together to exploit our knowledge of the radiation environment in the ISS, we need to prepare consistent sets of data.

This is an issue of importance when preparing databases to be used especially for radiation model validations. If, from one side, it is important to provide in these databases the least manipulated data to avoid contamination with uncontrollable procedures, it is also important, from the other side, to merge in a larger dataset consistent contributions from different devices, to be provided for model validation.

A corrected data set, which takes into account the limitations of each device, should therefore be prepared for each detector projecting the results into the same parameter space, so that any model will be able to use all data without further manipulation.

It is also apparent that this strategy will provide quite useful inputs for a deeper and better understanding of the compared detectors.

\subsubsection{ALTEA}

All geometrical issues, including the telescope features, are taken into account by the geometrical factor (GF). The electronic readout system of ALTEA, however, is strongly affecting the acceptance window of the device. As mentioned above, only ions releasing at least $3 \mathrm{keV} / \mu \mathrm{m}$ (in Silicon) can be measured, however to trigger the device the ion must release at least this quantity on all the $Y$ planes (i.e., the planes striped in the $Y$ direction are the trigger planes). Three "blind" areas are therefore observed:

1. the ion has an input energy $E_{\text {in }}$ too low to reach the last triggering detector plane, and it stops before (stopping ions);

2. the ion releases an amount of energy on a trigger plane which is below measurement threshold, in the ALTEA case $\approx 3 \mathrm{keV} / \mu \mathrm{m}$ (these are mostly protons);

3. the energy release of the ion [on the last plane(s)] is higher than the saturation, in the ALTEA case$\approx 700 \mathrm{keV} / \mu \mathrm{m}$ (saturating ions). Note that this case is not a real blind area: the ion is counted however the energy release is underestimated (and consequently its input energy $E_{\text {in }}$ is overestimated and/or the charge $Z$ underestimated).

Quantifying this effect provides the mean to correct the data accordingly.

A model of the GCR environment inside the ISS is provided by Cosmic Ray Effects on Micro-Electronics (1996 Revision) (CREME96; https://creme.isde.vanderbilt.edu). In a previous investigation (La Tessa et al. 2009) we fitted measured radiation values with a model based on total and partial charge-changing cross-sections and found an equivalent shielding of $5 \mathrm{~cm}$ of aluminum. We therefore used this value to model the radiation inside the ISS. The radiation due to 
Flux in ISS (noSAA, $\left.13.5 \mathrm{~g} / \mathrm{cm}^{\wedge} 2\right) \log \left\{\mathrm{n} / \mathrm{cm}^{\wedge} 2 \mathrm{sr}\right.$ s)], whole orbit

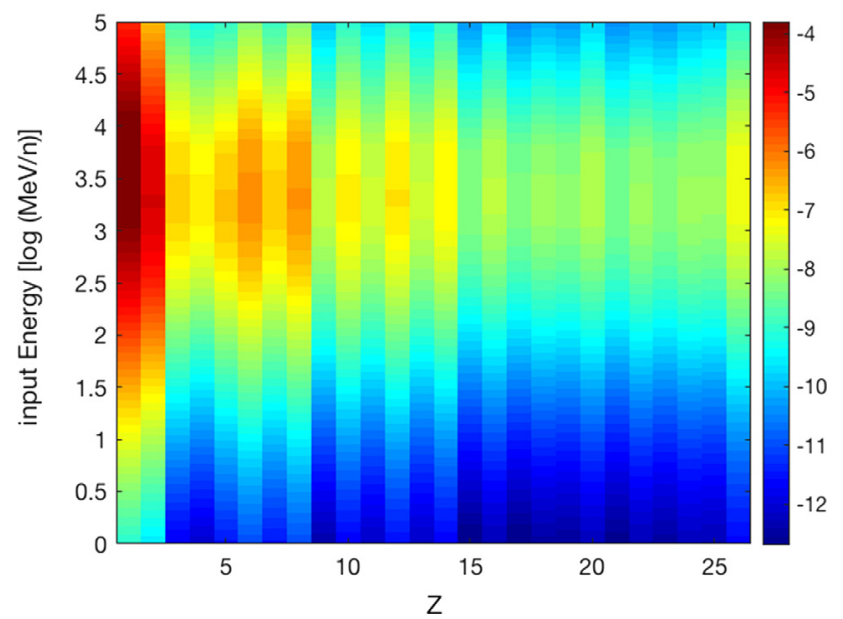

Fig. 4. Radiation flux in the ISS from CREME96.

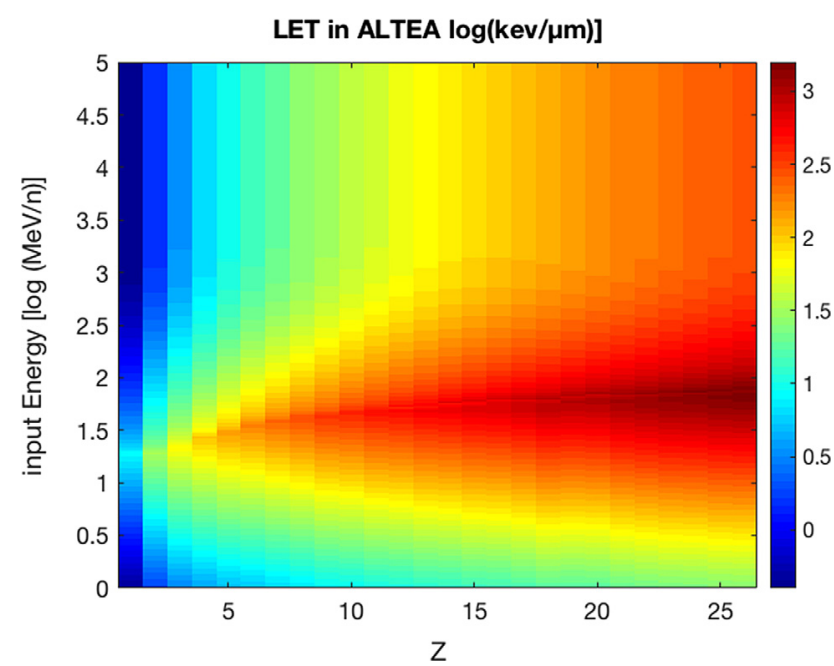

Fig. 5. Mean over the six planes of the LET in ALTEA.

GCR in the ISS is shown in Figure 4, where the logarithm of the flux is color coded against the logarithm of the input particle energy and its charge $Z$.

The ALTEA geometry has been therefore simulated using PHITS, generating a look-up table that provides the LET released in each of the six detector planes of one SDU, for each charge $Z$ and input energy $E_{\text {in }}$ (the average over the six planes is shown in Fig. 5). The element-by-element product of Figures 4 and 5 provides the LET that an ideal ALTEA should measure under the influence of the radiation environment simulated by CREME96 (the average over the six planes is shown in Fig. 6).

Taking this file as the reference, to consider points 1-3 above, we can write:

1. Stopping ions: LET $<0 \mathrm{keV} / \mu \mathrm{m}$ on the 5 th plane. ${ }^{3}$

2. Fast ions (non-triggering): LET $<3 \mathrm{keV} / \mu \mathrm{m}$ on any trigger plane.

\footnotetext{
${ }^{3}$ Operationally LET $=0$ means LET $<3 \mathrm{keV} / \mu \mathrm{m}$, however, in this case the ion does not even reach the plane. Fifth plane from the entrance, in the hypothesis that the ion enters from the first $Y$ plane (trigger plane).
}

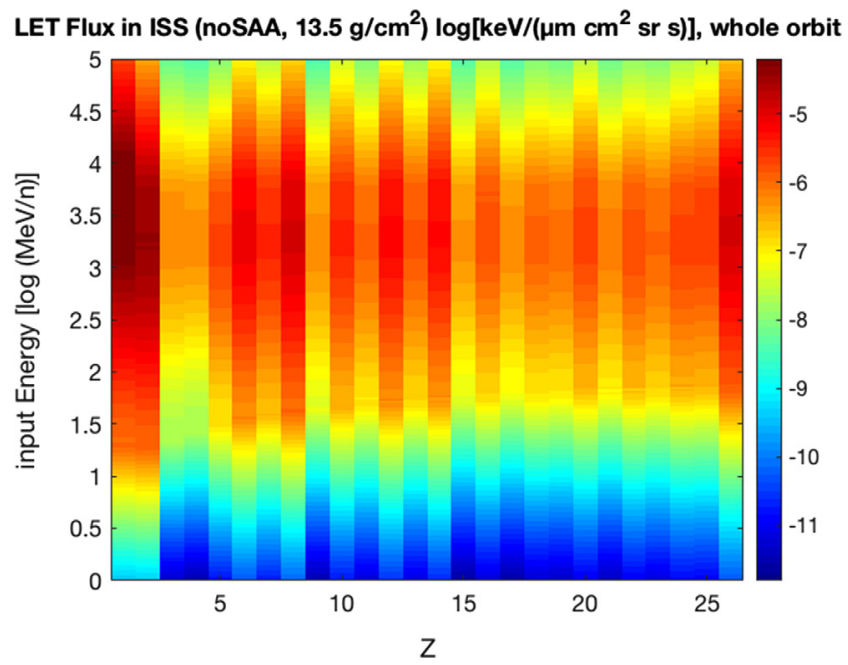

Fig. 6. LET measured by ALTEA in the ISS (following the GCR CREME model).

\section{Saturating ions: LET $>700 \mathrm{keV} / \mu \mathrm{m}$ on any plane.}

For each of the above conditions one matrix is built with a 1 for each element when the condition is not met and a 0 when it is met. A final element-by-element product of the three matrices produces a "Mask" showing what of Figure 6 is measured by ALTEA (corresponding to the ones), and what is not (zeros) due to the triggering and sensitivity characteristics of the detector.

A sum over all the elements of the "measured" matrix over the "ideal" matrix (Fig. 6) is providing an estimate of the portion of the total LET (or dose) measured by the ALTEA system $(41.2 \%)$. Repeating for the flux (directly from Fig. 4) it is possible to calculate the same value for the flux (1.27\%).

Repeating this calculation for each $Z$ the following is found:

The above values quantify the limitations mostly in proton (and Helium) detection that has been mentioned in the description of the ALTEA apparatus.

The radiation spectral composition varies across latitudes (and therefore $L$ values ${ }^{4}$ ). The percentage of radiation measured by ALTEA varies accordingly. Repeating the same above procedure for $27 L$ shells from $L=1.0 \pm 0.1$ to $L=6.2 \pm 0.1$ it is possible to generate a vector describing how much of the flux or of the dose is measured in each $L$ shell. Flux vector goes from a minimum of $1 \%$ to a maximum of $1.4 \%$, while dose vector from $39 \%$ to $46 \%$, consistently with the above values, calculated over all $L$ shells.

The final normalization of the data is then performed projecting these correction factors (function of $L$ ) to time, using the NASA provided ancillary data, and multiplying the original flux and dose data in time by the inverse of these projected factors.

\subsubsection{DOSTEL}

For the DOSTEL data the primary dataset (as given in the nominal dose rate and count rate mode) is taken as the baseline data for further data evaluation.

\footnotetext{
${ }^{4} L$ : McIlwain magnetic coordinate, McIlwain 1961
} 


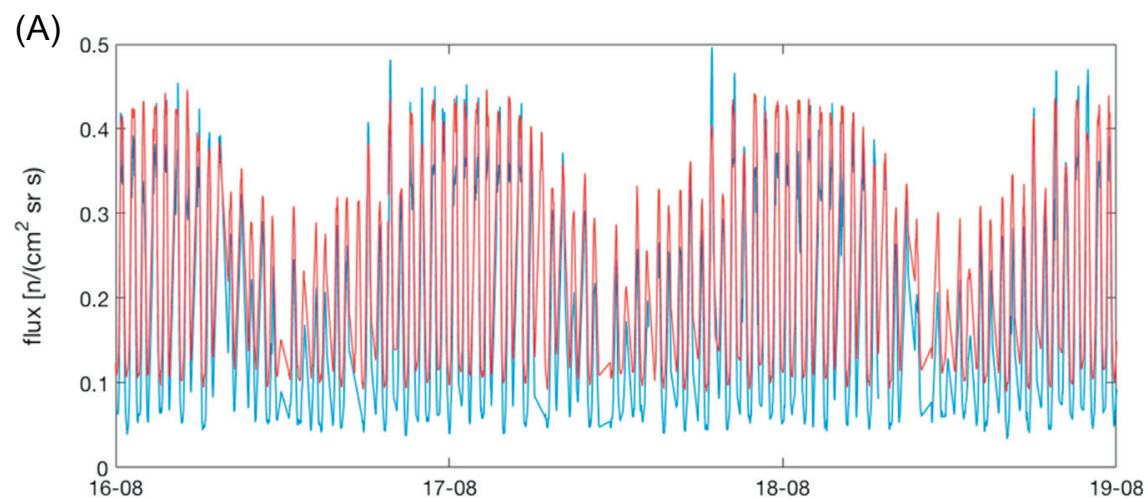

(B)

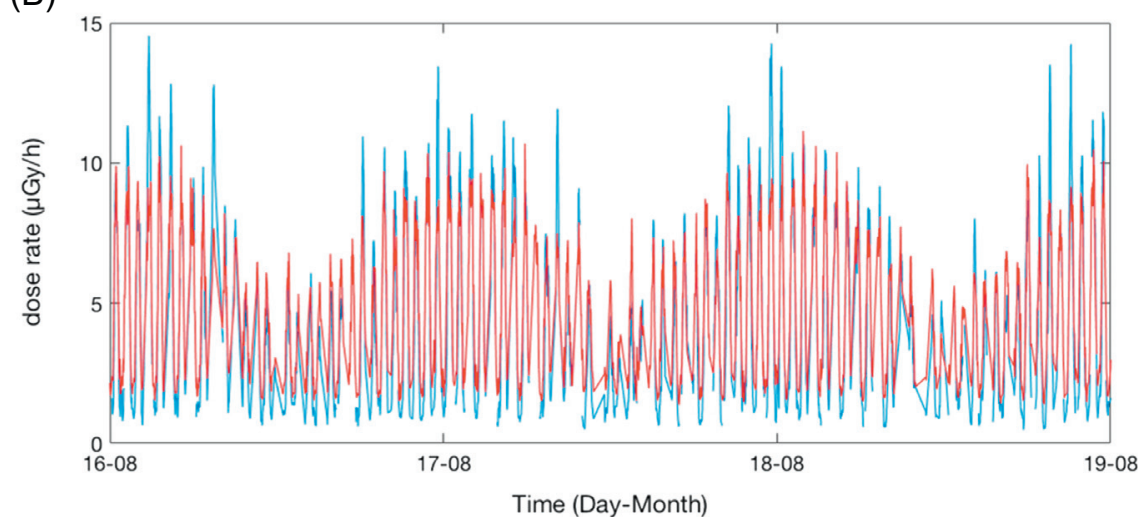

Fig. 7. Flux (A) and dose rate (B) of ALTEA (blue) and DOSTEL2 (red) during three days in the sample period. GCR data (SAA data have been removed).

\subsubsection{Time adjustment}

Once the two datasets have been normalized and made consistent with each other, it is useful to join the two datasets in a single matrix showing at each time point the output of the two devices as well as all the related ancillary data. The only issue here is to have data at the same time points, possibly equally spaced. In our case the data from ALTEA were minute-averaged while DOSTEL featured two different averaging times: $20 \mathrm{~s}$-averaged for the SAA passages and 100 s-averaged for the GCR. We decided to build a minuteaveraged matrix and consequently we interpolated DOSTEL data, using linear interpolation. A matrix is therefore built, each row representing one-minute point and the 33 columns containing all the flux and dose values measured by ALTEA in the three directions and by the two DOSTEL detectors, as well as all the relevant ancillary data (altitude, latitude, longitude, McIlwain $L, B$ (magnetic field), and rigidity).

\section{Results}

The ALTEA data normalized as described above, is finally used for comparison with the DOSTEL data. In the following the contribution from the SAA is removed using the magnetic coordinates: $B$ (magnetic field) and $L$ (the McIlwain coordinate, McIlwain 1961). In this work the SAA region is considered to be within $L<2.4$ and $B<0.27 \mathrm{G}$.

\subsection{Sample in quiet period}

A sample period was defined, from August 10th 2012 to August 31st 2012. This period featured no prominent solar activity, and during this time both ALTEA and DOSTEL (1 and 2) were active and in Columbus. ALTEA featured a single SDU, aligned along $Z$ and the two DOSTELs were, respectively, along $X$ and $Y$. The ALTEA SDU was inserted in an empty double drawer in ER3, while the two DOSTELs were positioned nearby beneath the EPM rack. Figure 7 shows a first comparison for flux (Fig. 7A) and dose rates (in $\mathrm{Si}$, Fig. 7B) for three days during the test period.

Figure 8 shows a dose rate comparison between the two DOSTELs and ALTEA using data from the whole test period.

Changing the amount of shielding in the CREME96 simulation pushes the above results further from the identity line. The chosen value $\left(13.5 \mathrm{~g} / \mathrm{cm}^{2}\right)$ appears therefore as an "optimal" value for this comparison. Finally Figure 9 shows the ratio between DOSIS and ALTEA dose rate values, against the McIlwain coordinate $L$.

\subsection{Entire period comparison}

The discrepancies between the ALTEA and DOSTEL datasets at low dose rates (low $L$ ), that can be recognized especially from Figure 7, are clearly visible over the whole studied periods (2010-2012) with about the same magnitude. The differences at high dose rates (high $L$ ) are, on the contrary, modulated by the ALTEA position and depend on the chosen telescope direction.

A whole period of comparison of flux and dose rates provides the results (at high latitude as selected from $L>3$ condition) shown in Figures 10 and 11, respectively, where the day-averaged results are shown between September 2010 and September 2012. 


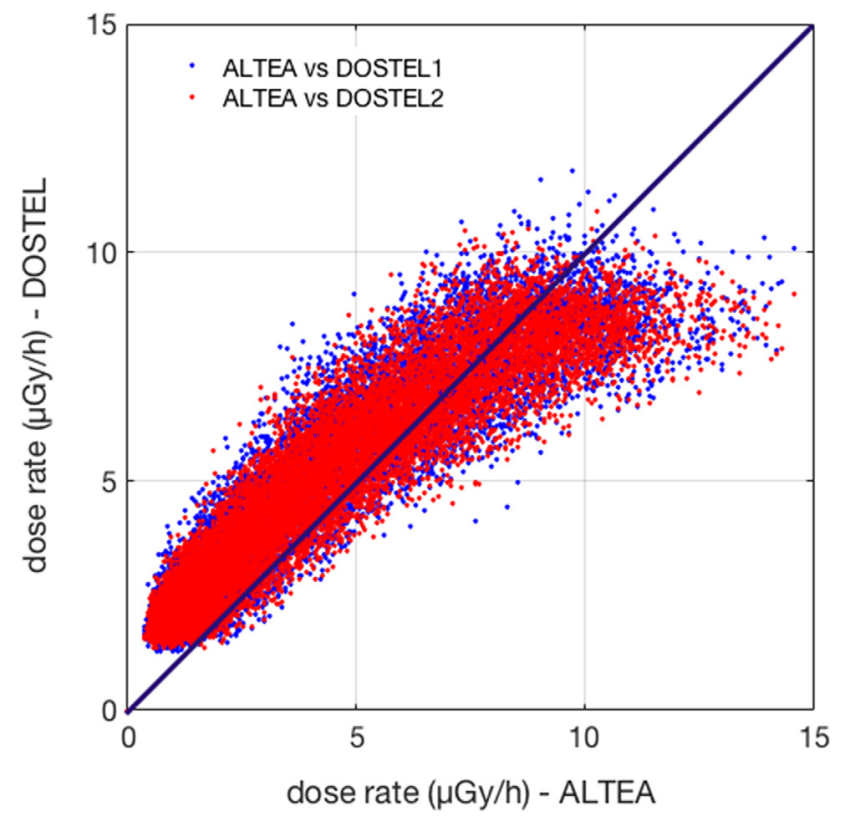

Fig. 8. Comparison between the ALTEA dose rate in $Z$ (abscissas) and DOSTEL-1, in $X$ (blue dots) and DOSTEL-2, in $Y$ (red dots). The line is the identity line and serves as eye guidance. Note: GCR data (SAA data have been removed).

Averaging the data in Figure 11 for each position of ALTEA, the mean dose rates can be calculated (Fig. 12).

\section{Discussion}

The aim of this paper is to present a procedure for the integration in a single database of radiation measurement results from different active detectors, and to illustrate the advantages of this merging in terms of data exploitation.

With two datasets coming from two different detector systems in general we have two possible approaches. The first is to reduce the data from the most sensitive detector systems to provide a homogeneous sensitivity for the input parameters. In the presented case the major "sensitivity" difference between the two analyzed systems is the lower threshold of the LET acceptance: $3 \mathrm{keV} / \mu \mathrm{m}$ for ALTEA and $0.16 \mathrm{keV} /$ $\mu \mathrm{m}$ for DOSTEL. It could be suggested, therefore, to cut the DOSTEL data from $3 \mathrm{keV} / \mu \mathrm{m}$ and compare and merge the two datasets in this way. The second approach, the one followed in this paper, starts with the definition of a target "sensitivity", in our case the $100 \%$ of the impinging ions, requires models to calculate the missing part on a detector, and to re-normalize the output of the detector.

Pros and cons of the two approaches are evident. With the first we have the least (possibly even none) manipulation of the data, but the approach is meaningful only with two (or a very small number) of datasets. Using this approach with many detector systems would degrade the sensitivity of the merged datasets to the lowest sensitive detector, probably losing a large amount of information, and, furthermore, may require further degrading when a new dataset from a different detector is added. Conversely the second approach provides a "frame" good for all detectors, without loss of any information. This is at the expense of the added data manipulation, and of the dependency (at least in part) of the final dataset on the

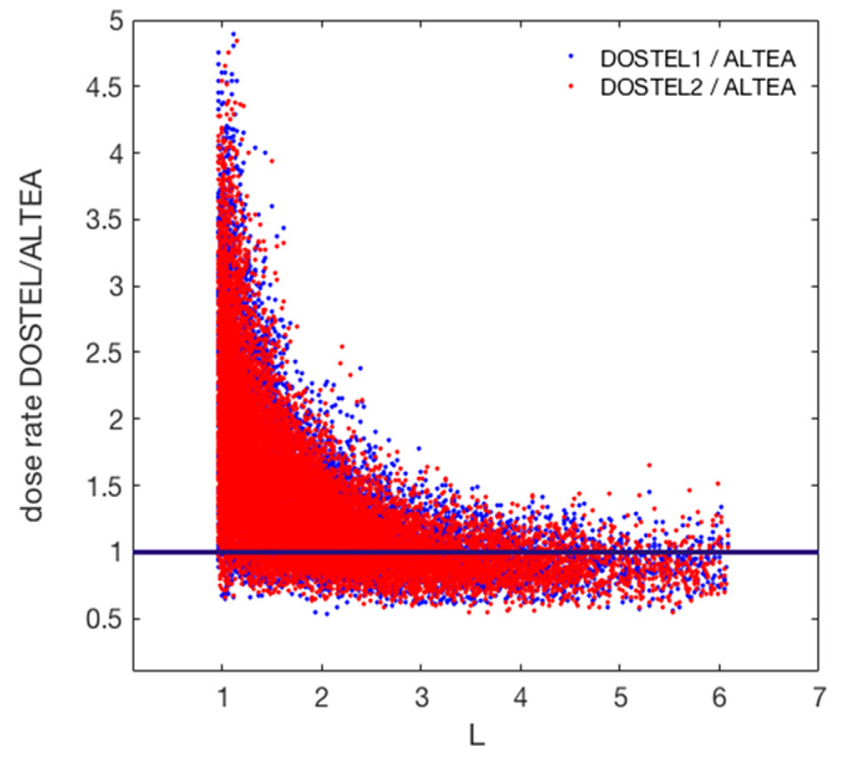

Fig. 9. The ratio between DOSTEL1 and ALTEA dose rate measurements (blue dots) and between DOSTEL-2 and ALTEA dose rate measurements (red dots) vs. McIlwain coordinate $L$, in the test period. Note: GCR data (SAA data have been removed).

chosen model used to calculate the missing data for some of the detectors.

We chose the second approach using, as model, CREME96. This model choice is strongly motivated by the wide availability of the model itself and supported by the good agreement found between the re-normalized ALTEA data and the DOSTEL data. Weaknesses of the CREME96 model have been nevertheless pointed out in recent works (see, e.g., Mrigakshi et al. 2012). Further work is in progress to test the sensitivity of this re-normalization on the chosen model, by using more performing tools such as Badhwar/O'Neill (O’Neill et al. 2015) or DLR (Matthiä et al. 2013) models.

As mentioned the agreement between our two datasets is quite convincing. However, the comparison shows also a few discrepancies [low latitude (low L) ALTEA excess, high latitude (high $L$ ) DOSTEL saturation] that we will now discuss in more detail.

The low latitude excess is about constant over the whole period of comparison and also when considering different directions. The absence of correlation with the position in the ISS and the direction leads to a lack of dependence on the amount of shielding, including Earth shielding. We also considered noise contribution in DOSTEL (rising the counting at the lowest rates) but this can be excluded due to the DOSTEL electronics design. As shielding effects are ruled out (see above) we considered the possible contribution of secondary $e, e^{+}, \pi$, and $\mu$, measured by DOSTEL but not by ALTEA. However, a simple calculation based on Slaba et al. (2013) provided an approximate set of values for this correction and (i) this is a much larger correction for high $L$ than low $L$ (due to the higher ion flux), (ii) in any case the correction is small enough not to be able to correct the discrepancy at low $L$.

The effects of this low $L$ excess on the daily averaged data are minimal as the flux (and dose rate) is minimal at low latitude due to the high magnetic cut-off rigidity (low $L$ ). So we left open this issue with the understanding that it does not modify significantly the daily averages. 


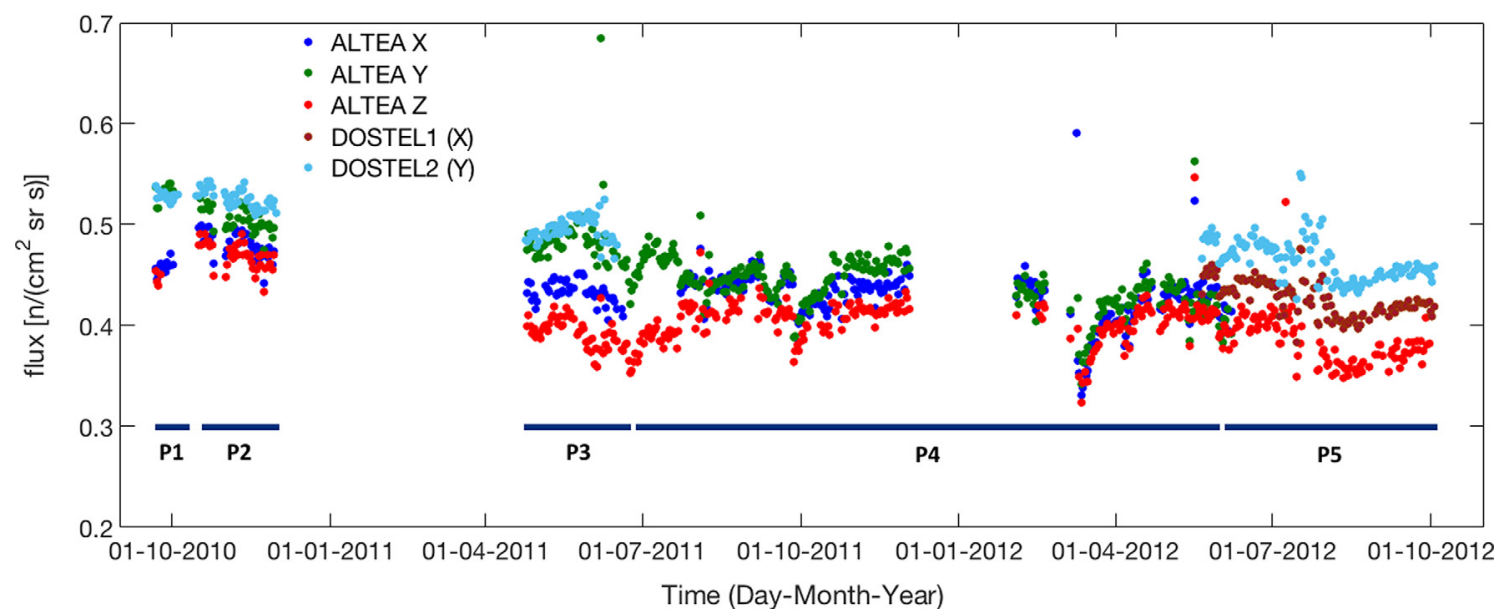

Fig. 10. Flux as measured by ALTEA and the two DOSTELs over the whole investigated period. McIlwain $L>3$. Data are averaged over each day. In the bottom, the indication of the positions of the ALTEA system is shown (see also Fig. 1). The March 2012 SPE has been cut to improve readability of the rest of the data. Note: GCR data (SAA data have been removed).

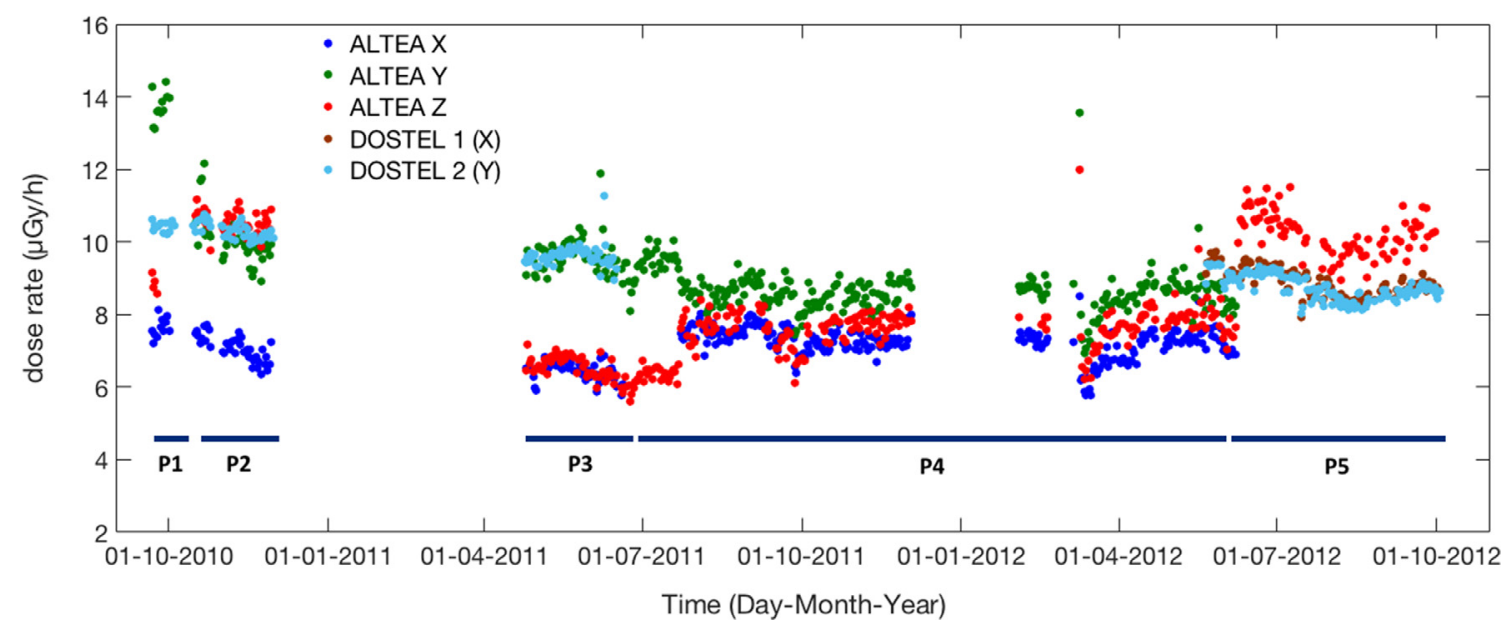

Fig. 11. Dose rate as measured by ALTEA and the two DOSTELs over the whole investigated period. McIlwain $L>3$. Data are averaged over each day. In the bottom, the indication of the positions of the ALTEA system is shown (see also Fig. 1). The March 2012 SPE has been cut to improve readability of the rest of the data. Note: GCR data and SAA data have been removed.

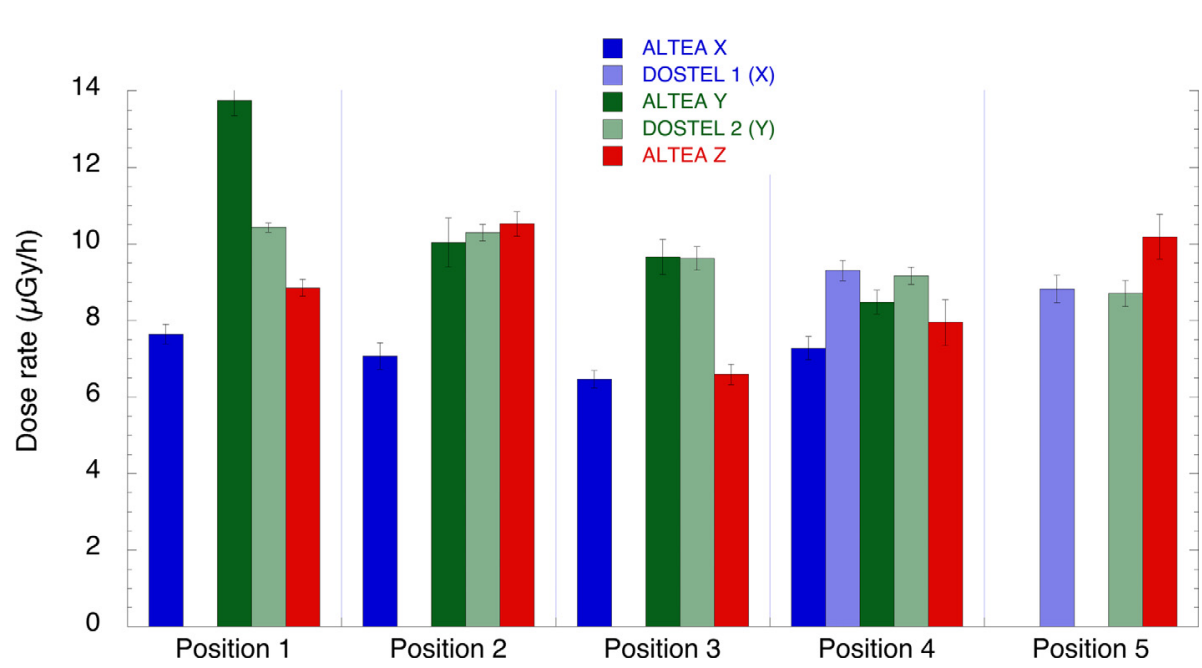

Fig. 12. Dose rate as measured by ALTEA and the two DOSTELs over the whole investigated period, averaged for each position of ALTEA. Mcllwain $L>3$. The contribution of March 2012 SPE is selected out. Darker: ALTEA; lighter: DOSTEL. Note: GCR data and SAA data have been removed. 
Table 2. Percentage of flux and dose rates measured by ALTEA for different ions (see text).

\begin{tabular}{ccc}
\hline \hline$Z$ & Flux [\%] & Dose rate [\%] \\
\hline 1 & 0.024 & 0.29 \\
2 & 3.2 & 8.7 \\
$>2$ & $>98 \%$ & $>94 \%$ \\
\hline
\end{tabular}

The high latitude saturation is not related to the value used for the shielding in the CREME96 model. Varying the shield produces a rotation of Figure 8 centered approximately on the lowest dose points (clockwise for increasing shielding, counterclockwise for decreasing shielding). Also the width of the plot slightly decreases with increasing shielding. The saturation remains and, using a different value than $5 \mathrm{~cm}$ of aluminum, the average distance from the identity line increases, somehow suggesting that the chosen equivalent thickness is close to optimal. The saturation is also not due to the interpolation of the DOSTEL data, as different interpolating algorithms as well as a totally re-built matrix with only selected data (taking one out of six points of ALTEA and one out of three points of DOSTEL, with no interpolation) showed no difference in the saturation behavior. While singularly those points represent the higher dose values, they are reached rarely, so even this discrepancy does not show up in the daily average. With this understanding we left also this issue open.

When ALTEA was in P1, P2, P3 only DOSTEL-2 was "on". A remarkable agreement is shown (Figs. 10 and 11) between DOSTEL-2 $(Y)$ and ALTEA $Y$ in positions P2 and P3. ALTEA reported a much higher dose level in P1. Looking at the positions of the devices (Fig. 1), ALTEA $Y$ sees, beside hull and racks, partly the ISS truss (especially in P2 and P3) and DOSTEL-2 sees along the Columbus/JEM (Japanese Experiment Module) modules, but also, due to the large acceptance angle, directly across the Columbus hull. The very similar dose values could suggest that the two average shieldings seen by ALTEA $Y$ and DOSTEL-2 $(Y)$ while in P2 and $\mathrm{P} 3$ are not significantly different. In P1 ALTEA was in an empty rack so it looked only at the hull of the module: the lower shielding in this site is causing the higher dose measured by ALTEA.

The second period of comparison is when ALTEA was in Position P5. In this period, we have "on" one ALTEA $Z$ detector and both DOSTEL-1 $(X)$ and DOSTEL-2 $(Y)$. The dynamic of the flux and dose rate behavior is quite similar for the three detectors; however, the averages feature intriguing characteristics. The dose rates measured by the two DOSTELs are about equal, but the corresponding fluxes in $Y$ are consistently higher than those in $X$. Furthermore, the $Z$ values, measured by ALTEA, are the lowest one in flux and the highest one in dose rate. Let us consider the two DOSTEL measurements in P5 and the measurements ALTEA $X$ and $Z$ in P3. Also in this last case the dose rate values are almost coincident while the ALTEA $X$ fluxes are consistently higher than the ALTEA $Z$ fluxes. This can be explained (Narici et al. 2015a) taking into account the different amount of shielding: in P3, in $X$, there is much more shielding than in $Z$. Therefore, in $X$ there is more fragmentation (higher flux) and consequently a lower dose per ion providing grounds for the similar values in dose rates. Applying the same argument to the two DOSTEL measurements in P5 would require $Y$ to have more shielding than $X$. And, indeed, in Columbus this is the case as the two axes have inverted characteristics: $X$ is looking directly outside Columbus and features surely less shielding than $Y$, that looks through the long "Columbus - Node - JEM" train of modules. The understanding of the ALTEA measurements in Columbus requires a further consideration about the peculiar position inside the ER3 rack. The fields of view of the detector are fully covered by the above and below drawers/experiments. The spectra of the $Z$ data in this position (Narici et al. 2015a) shows higher (than in the other positions) flux for all LET values above carbon, and lower below. The higher dose rate in $Z$ shown in Figure 11 is therefore probably an effect of a different radiation quality due to a significant amount of higher LET ions possibly generated by the interactions of the primary radiation with the material closely surrounding ALTEA.

It is apparent that this comparison lacks a baseline concurrent measurement with the two instruments in similar position (same shielding in the fields of view) and at the same time. This unfortunately never happens. A suggestion would be to make mandatory a short baseline measurement for each new uploaded detector in the ISS with some sort of standard detector so as to provide a reliable cross-calibration. Understanding the dynamics of the detectors is what we can do with the existent data (as we did in this work). This is important because we can use together the data for model validation and testing. However, only an initially properly conducted cross-calibration would fully exploit the value of the detectors measurements.

Considering all presented data the importance of spacecraft shielding (hull and equipment inside) becomes overwhelming. The data suggest higher radiation quality (and therefore risk) closeby racks due to target fragmentation as well as a confirmation of strong radiation asymmetries due to the shape of the ISS as well as to its content.

The method to build a common data matrix from two different devices appears to be solid. Together with metadata information about the detectors, this joint data matrix, easily expandable to many devices, could be an extremely useful data package for model testing and validation.

Acknowledgements. DOSIS: The CAU, University of Kiel was supported by DLR under Grants 50WB0826, 50WB1026, $50 \mathrm{WB} 1232$, and 50WB1533. The authors would like to thank the HIMAC team at NIRS in Japan for providing lots of experiment time. The authors gratefully acknowledge the support of the European Space Agency (ESA), especially Jason Hatton, Rene Demets, Chiara Lombardi, and Liesbeth De Smet as well as colleagues from CADMOS (Lourdes Oro Marot, Cécile Thevenot) Toulouse, France, DLR-MUSC (Pascaline Kerbeci), Cologne, Germany, and ESA-EAC, Cologne, Germany. All of these experiments would have not been possible without the help of all the astronauts working on the DOSIS and DOSIS 3D experiments.

ALTEA: The authors acknowledge the support from ESA (programs ALTEA-shield) from ASI, that made the development of the ALTEA detector possible (Contracts ALTEA, MoMa-ALTEA, and ALTEA-support). The authors would also like to thank Dr René Demets from ESA for his very valuable support during the ALTEA-shield project. Finally, the authors are thankful to the ISS crew members who made all of this possible.

The editor thanks Francis Badavi and an anonymous referee for their assistance in evaluating this paper.

\section{References}

Benton, E.R., E.V. Benton, and A.L. Frank. Conversion between different forms of LET. Radiat. Meas., 45(8), 957, 2010, DOI: $10.1016 /$ j.radmeas.2010.05.008. 
Berger, T., S. Burmeister, M. Matthiä, B. Przybyla, G. Reitz, et al. DOSIS \& DOSIS 3D: radiation measurements with the DOSTEL instruments onboard the Columbus laboratory of the ISS in the years 2009-2016. J. Space Weather Space Clim., 7, A8, 2017, DOI: $10.1051 /$ swsc/2017005.

Berger, T., B. Przybyla, D. Matthiä, G. Reitz, S. Burmeister, et al. DOSIS \& DOSIS 3D: long-term dose monitoring onboard the Columbus Laboratory of the International Space Station (ISS). J. Space Weather Space Clim., 6, A39, 2016, DOI: $10.1051 / \mathrm{swsc} / 2016034$

Di Fino, L., F. Belli, V. Bidoli, M. Casolino, L. Narici, et al. ALTEA data handling. Adv. Space Res., 37, 1710-1715, 2006 DOI: $10.1016 /$ j.asr.2005.01.105.

Di Fino, L., V. Zaconte, M. Ciccotelli, M. Larosa, and L. Narici. Fast probabilistic particle identification algorithm using silicon strip detectors. Adv. Space Res., 50, 408-414, 2012, DOI: $10.1016 /$ j.asr.2012.04.015.

Durante, M., and F.A. Cucinotta. Physical basis of radiation protection in space travel. Rev. Mod. Phys., 83, 1245, 2011, DOI: 10.1103/RevModPhys.83.1245.

La Tessa, C., L. Di Fino, M. Larosa, L. Narici, P. Picozza, and V. Zaconte. Estimate of the space station thickness at a USLab site using ALTEA measurements and fragmentation cross sections. Nucl. Instrum. Methods Phys. Res., Sect. B, 267, 3383-3387, 2009, DOI: 10.1016/j.nimb.2009.06.107.

Matthiä, D., T. Berger, A.I. Mrigakshi, and G. Reitz. A ready-to-use galactic cosmic ray model. Adv. Space Res., 51, 329-338, 2013, DOI: $10.1016 /$ j.asr.2012.09.022.

McIlwain, C.E. Coordinates for mapping the distribution of magnetically trapped particles. J. Geophys. Res., 66, 3681-3691, 1961, DOI: 10.1029/JZ066i011p03681.

Mrigakshi, A.I., D. Matthiä, T. Berger, G. Reitz, and R.F. Wimmer-Schweingruber. Assessment of galactic cosmic ray models. J. Geophys. Res., 117, A08109, 2012,

DOI: $10.1029 / 2012 J A 017611$.
Narici, L. Heavy ions light flashes and brain functions: recent observations at accelerators and in spaceflight. New J. Phys., 10, 075010, 2008, DOI: 10.1088/1367-2630/10/7/075010.

Narici, L., M. Casolino, L. Di Fino, M. Larosa, P. Picozza, and V. Zaconte. Radiation survey in the International Space Station. J. Space Weather Space Clim., 5, A37, 2015a, DOI: $10.1051 /$ swsc/2015037.

Narici, L., T. Berger, D. Matthiä, and G. Reitz. Radiation measurements performed with active detectors relevant for human space exploration, Front. Oncol., 5, 273, 2015b, DOI: 10.3389/fonc.2015.00273.

O'Neill, P.M., S. Golge, and T.C. Slaba. Badwhar-O'Neill 2014 Galactic cosmic ray flux model description. NASA/TP2015-218569, 2015.

Slaba, T.C., S.R. Blattning, B. Reddell, A. Bahadori, R.B. Norman, and F.F. Badavi. Pion and electromagnetic contribution to dose: comparisons of HZETRN to Monte Carlo results and ISS data. Adv. Space Res., 52, 62-78, 2013, DOI: 10.1016/j.asr.2013.02.015.

Sullivan, J.D. Geometrical factor and directional response of single and multi-element particle telescopes. Nucl. Instrum. Meth., 95, $5-11,1971$.

Zaconte, V., F. Belli, V. Bidoli, M. Casolino, L. Di Fino, et al. ALTEA: the instrument calibration. Nucl. Instrum. Methods Phys. Res., Sect. B, 266, 2070-2078, 2008,

DOI: $10.1016 /$ j.nimb.2008.02.072.

Zaconte, V., M. Casolino, C. De Santis, L. Di Fino, C. La Tessa, M. Larosa, L. Narici, and P. Picozza. The radiation environment in the ISS-USLab measured by ALTEA: spectra and relative nuclear abundances in the polar, equatorial and SAA regions. Adv. Space Res., 46, 797-799, 2010a, DOI: 10.1016/j.asr.2010.02.032.

Zaconte, V., M. Casolino, L. Di Fino, C. La Tessa, M. Larosa, L. Narici, and P. Picozza. High energy radiation fluences in the ISS-USLab: ion discrimination and particle abundances. Radiat. Meas., 45, 168-172, 2010b, DOI: 10.1016/j.radmeas.2010.01.020.

Cite this article as: Narici L, Berger T, Burmeister S, Di Fino L, Rizzo A, et al. Exploiting different active silicon detectors in the International Space Station: ALTEA and DOSTEL galactic cosmic radiation (GCR) measurements. J. Space Weather Space Clim., 7, A18, 2017, DOI: $10.1051 /$ swsc/2017016. 